\title{
Probabilistic modelling of automotive body joints
}
A. S. Blicblau*
M. Singh*
B. Strahan*
C. Torpy*

(Received 6 October 2005; revised 30 November 2006)

\begin{abstract}
We investigate the manner in which variability in the quality of spot welds in automotive body joints contributes to variability in joint stiffness. Joint stiffness is defined here as the resistance to rotation of the joint. The aim is to develop a method of investigating problems of this nature using simulation and statistical techniques, and then to quantify the variation for this particular case. The results are validated by ensuring that the accuracy and resolution of the model are appropriate. Here, accuracy is the degree to which the model represents reality and is determined by achieving convergence of results as finer mesh densities are used.
\end{abstract}

${ }^{*}$ Faculty of Engineering and Industrial Sciences, Swinburne University of Technology, Hawthorn Australia. mailto:msingh@swin.edu.au

See http://anziamj.austms.org.au/V47EMAC2005/Blicblau for this article, (C) Austral. Mathematical Soc. 2007. Published March 5, 2007. ISSN 1446-8735 


\section{Contents}

1 Introduction

C571

2 Modelling automotive spot welds

C572

2.1 Method of moments . . . . . . . . . . . . . C573

3 Quantifying joint stiffness

C574

3.1 Loading case simplification . . . . . . . . . . . . C575

3.2 Modelling . . . . . . . . . . . . . . . C577

4 Validation

C580

4.1 Variation in joint stiffness . . . . . . . . . . . C581

5 Conclusions

C581

References

C583

\section{Introduction}

The manufacture of modern automotive body parts involves, amongst other processes, drawing thin sheet metal into curved shapes and spot welding the sections together to form thin walled tubes which have irregular cross sections. With the advent of computer aided engineering, the shape of the body has been greatly improved, while also becoming more complex. Because of this, research still needs to be conducted in the area of quantifying the effect of manufacturing tolerances on body performance.

The performance properties of a car's body structure can vary greatly with only a small variation in the construction characteristics of that structure. Of particular interest is the behaviour of the T-joint joining the roof sill (cantrail) to the centre pillar (B-C pillar) of a sedan-style automobile. It 
is often the case that the customer notices the variation in a product more than the performance of the product. It is therefore important to not only design for good performance but to also design for minimum variability.

Current standard practice in the automotive industry is to use a deterministic approach to design, with the Six Sigma approach being used to define the range of allowable tolerances for given components [1]. When designing automotive body joints, factors of safety are used to take into account weld variation. A reasonable approach would be to assume each weld is $80 \%$ effective. Making the reasonable assumption that Six Sigma Quality has been implemented, the standard deviation of weld quality, $\sigma$, is approximately $\sigma=(1-0.8) 6 \approx 0.0334$.

Monte-Carlo simulations of weld quality variations provide us with a technique for randomly generating large quantities of data that fit an experimentally determined distribution, which is the novelty of this paper. The six sigma value of $\sigma=0.0334$ has been used in the Monte-Carlo simulation.

We examine the joint stiffness and how it relates to variation in spot weld quality. We present a simulation model that closely relates to the physical welded T-joint, and show that the simulations performed represent a real world scenario. The model is validated and then analysed using these probabilistic design methods.

\section{Modelling automotive spot welds}

The most prevalent metal joining method in the automotive industry is resistance spot welding. The process involves passing a large current through two thin sheets pressed together at a predefined pressure. If the pressure is sufficient, the highest electrical resistance will be at the interface. The heat generated from the electrical resistance welds the sheets together. While resistance spot welding has many advantages, quality can be difficult to de- 
termine. Current practice in the automotive industry is to destructively test welds by separating the joined sheets. A weld is deemed to be acceptable if the backing sheet fails before the weld.

A major engineering problem that faces current automotive designers is how to accurately represent resistance spot welds in computer models for developing new elements. However, these models do not take into account random welding defects or inclusions, which have the effect of reducing the load carrying capacity of the weld. One possible approach is to use a reduced 'equivalent volume' for the weld to simulate the weakening effect of weld defect (Kuo et al. [5]) while still keeping the model simple. This approach is similar to the one used by Beer et al. [2] to analyse the bending of composite beams.

The spot-weld volume is modelled as a dimensionless 'volume'

$$
V_{*}=V / V_{\text {nom }},
$$

where $V$ is the equivalent weld volume and $V_{\text {nom }}$ is the nominal weld volume. The dimensionless quantity $V_{*}$ is a measure of the quality of the weld and varies from 0 (a weld with no strength) to 1 (a perfect weld) and higher (welds which are stronger than necessary). In the Monte-Carlo simulations $V_{*}$ is generated from a normal distribution with some specified mean and standard deviation.

\subsection{Method of moments}

While the Monte-Carlo method generates input data for the spot-weld volume, the Method of Moments is used to determine output characteristics of joint stiffness as given by Steele [9]. The Method of Moments assumes that there exists a function $g(x)$ that maps the input variable $V_{*}$ to the output variable $Y$. In our case the input variable is weld volume and the output variable is joint stiffness. The expectation operator $E$ is applied to $g(x)$ which 
is then expanded in a Taylor series about the mean of $x$. The mean of $Y$ is simply $E[g(x)]$ while the variance is $E\left[g(x)^{2}\right]-E[g(x)]^{2}$. Expressions for the mean and variance of $Y$ are presented in finite difference form without proof (Steele [9]).

$$
\begin{aligned}
& E\left[g\left(x_{1}, x_{2}, \ldots, x_{n}\right)\right] \\
& =g\left(\mu_{1}, \mu_{2}, \ldots, \mu_{n}\right)+\frac{1}{2} \sum_{i=1}^{n} \frac{g_{p-1}^{i}-2 g_{p}^{i}+g_{p+1}^{i}}{h^{2}} \nu_{i} \\
& E\left[\left(g\left(x_{1}, x_{2}, \ldots, x_{n}\right)-E\left[g\left(x_{1}, x_{2}, \ldots, x_{n}\right)\right]\right)^{2}\right] \\
& =\sum_{i=1}^{n}\left[\left(\frac{g_{p+1}^{i}-g_{p-1}^{i}}{2 h}\right)^{2} \nu_{i}+\frac{g_{p+1}^{i}-g_{p-1}^{i}}{2 h} \cdot \frac{g_{p-1}^{i}-2 g_{p}^{i}+g_{p+1}^{i}}{h^{2}} S_{i}\right. \\
& \left.+\frac{1}{4}\left(\frac{g_{p-1}^{i}-2 g_{p}^{i}+g_{p+1}^{i}}{h^{2}}\right)\left(K_{i}-\nu_{i}^{2}\right)\right] .
\end{aligned}
$$

The mean and variance of the input variables $x_{i}$ are represented by $\mu$ and $\nu$ respectively. In the case of spot-weld simulation there would be one $x$ for each spot weld each having the same PDF. The above formula is a simplification that neglects covariance between spot welds because we assume that the weld quality is independent of previous welds; this property is assumed throughout this paper. The derivatives in (2) and (3) are approximated using the finite difference approximations given by Burden \& Faires [3].

\section{$3 \quad$ Quantifying joint stiffness}

There are many different methods for quantifying the stiffness of an automotive body joint. A common approach used in industry is to construct a joint stiffness matrix (Okabe \& Tomioka [6]). The process involves defining the stiffness as the resistance to displacement to a force in the same direction. In matrix form the relationship is

$$
[F]=[K][x],
$$


where the matrix $[F]$ contains forces in any given direction $\left(F_{x}, F_{y}\right.$ or $\left.F_{z}\right)$, $[K]$ is the stiffness matrix and $[x]$ is the corresponding displacement matrix. This formulation can be naturally expanded to cover an arbitrary number of joints by extending the matrix $[K]$.

Many alternative approaches do exist to quantify the joint stiffness. For example, Song et al. [8] analysed the stiffness of an entire body-in-white (car body) as opposed to a single joint. The method employed in this paper was developed to gain maximum insight into the variation of joint stiffness at a single location. The joint stiffness was evaluated by calculating the rotation (about the centre point) of each point along the centre line.

\subsection{Loading case simplification}

The largest forces that are experienced in an automobile, excluding a crash, are when the wheels hit an obstacle at speed: for example, a gutter or speed hump, or when the car is dropped from a height onto all four wheels such as when the car 'lands' after going over a railway crossing. These forces are applied through the wheels and result in a system of compressive and tensile forces running through the chassis and frame. The loading case we are using is of the latter type, when a load is applied vertically through all four wheels at the same time.

Fenton [4] states that in the loading case (in a standard sedan) the forces act as a pair of couples around the centre of gravity of the car when we simplify the car to 2D. This produces compression in the roof and tension in the floor members. Also, due to the positioning of the centre pillar with respect to the front and back wheels, under this loading case there will be some bending in the pillar towards the front of the car. We chose to isolate the joint itself, so the loading case used has compressive forces acting through the horizontal members of the T-joint and a moment applied in the plane of the joint about the vertical member. 


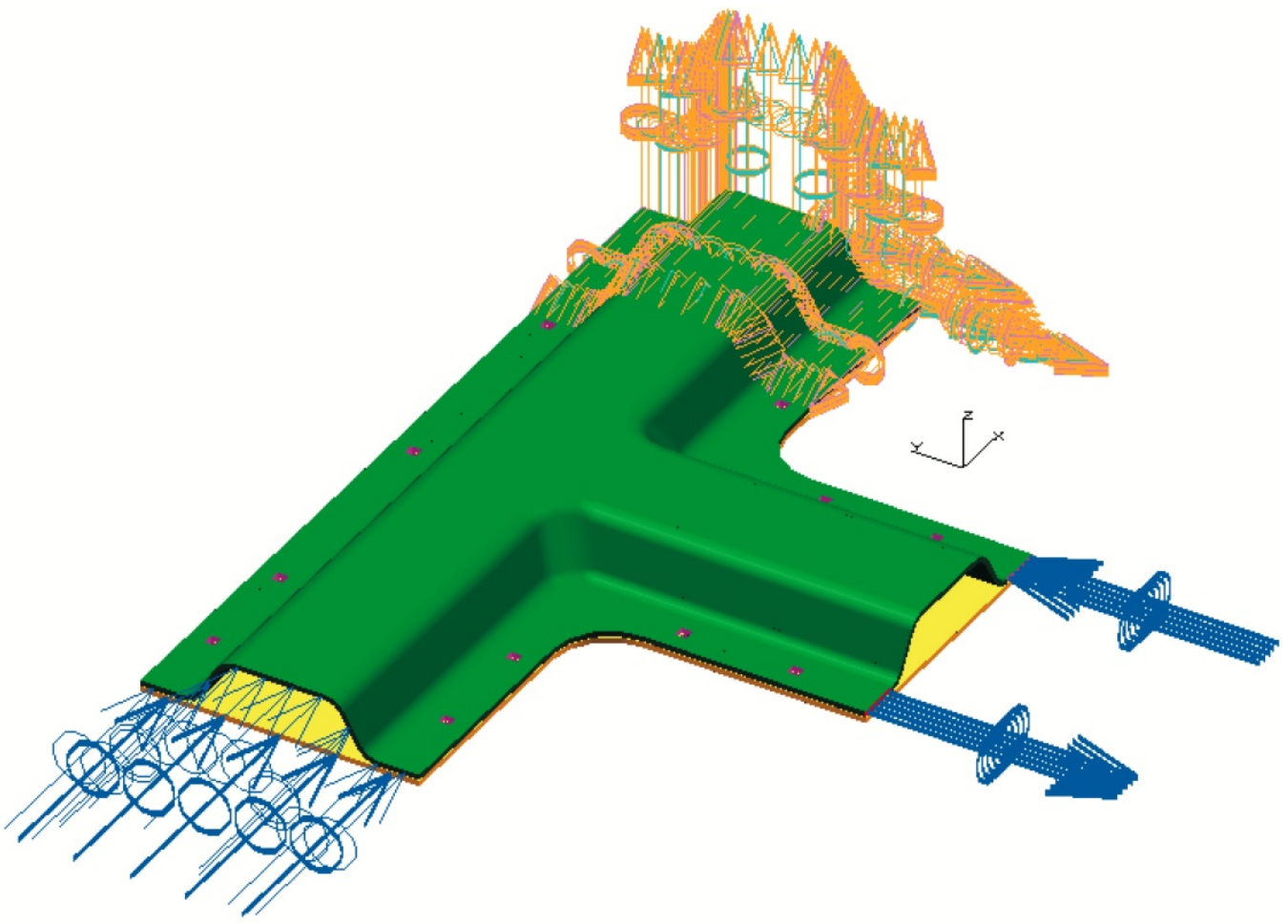

FiguRE 1: Load and boundary conditions 
The loading and boundary conditions are shown in Figure 1. The upper right face, when viewed down the $z$-axis of Figure 1, is restrained in all translational modes of freedom. Pressures are applied to the lower extremities of the faces at the flanges to simulate the moment about the $z$-axis.

The usual shapes for the cross section of car body members are quite complex. They typically have ribs and folds built in for rigidity and large radii to reduce stress concentration. These are constructed by stamping out the basic shapes and then spot welding the parts together to form a tubular assembly.

Fenton [4] also states that while these shapes have obvious structural benefits over basic tubes and channels, they can nevertheless be represented by fairly simply cross sections. The assumption must be made that the value of $I$ (moment of inertia) in the simplified model is equivalent to the real $I$ for the method to be valid.

The sharp corners and bends were replaced by gently curved radii to remove stress concentrators. Our intention was to measure the stress that passes through the spot welds, and these concentrators could possibly have introduced unwanted variation. Our solid model was created in IDEAS using two extrusions of the cross section which were melded together using the 'fillet' command to create a smooth transition.

\subsection{Modelling}

The first model, called the single solid model, was created in IDEAS and exported as a single solid in IGES (International Graphical Exchange System) format. There was a $1 \mathrm{~mm}$ gap between the upper and lower plates, and the welds were represented by extrusions that bridged the gap. This was meshed using an automatically generated solid 8 node element in ANSYS and the results are given in Table 1 . The mesh generator in ANSYS automatically constructs very dense meshes. 


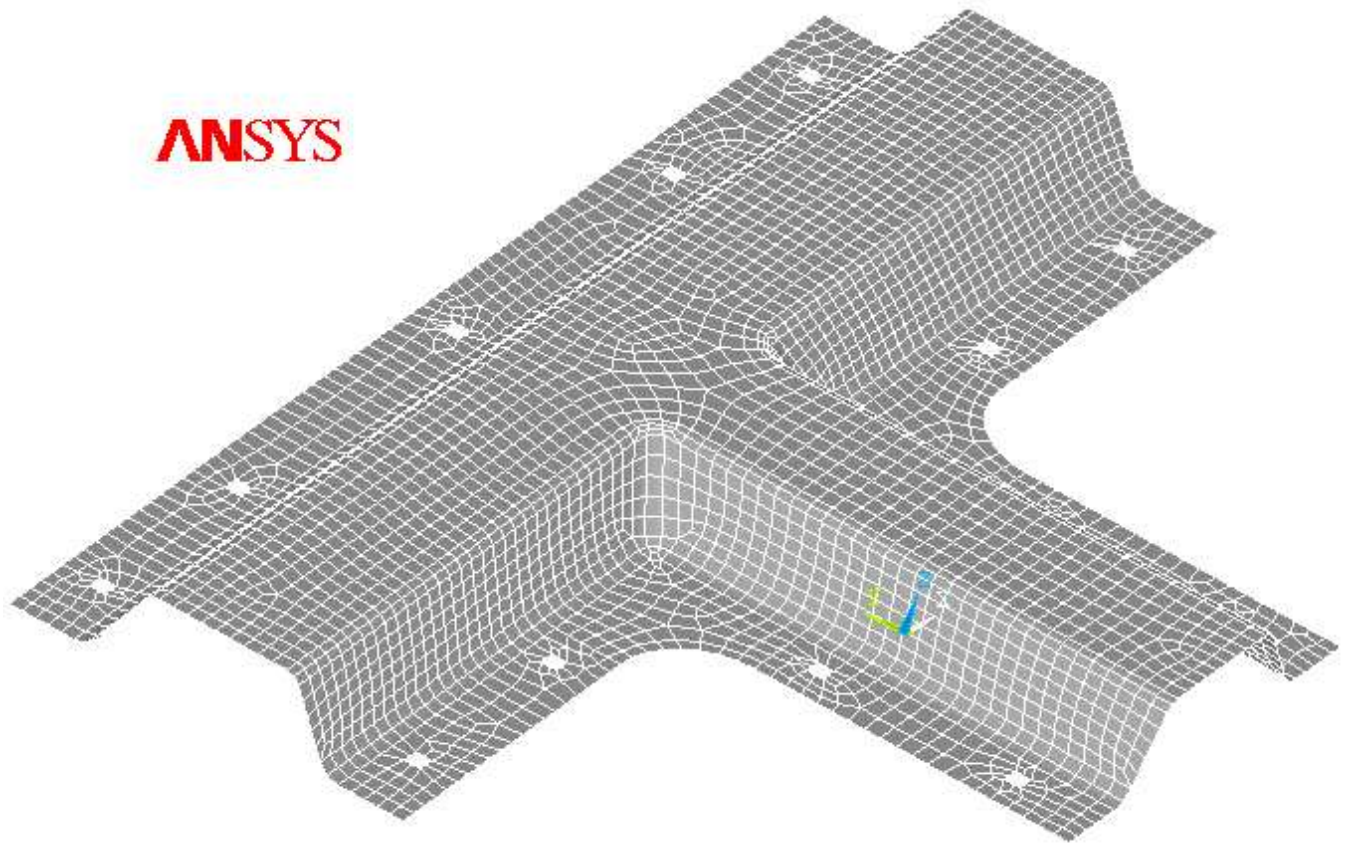

FIGURE 2: Upper surface of the shell mesh (lower surface removed for clarity)

TABLE 1: Convergence of results for single solid with gap.

\begin{tabular}{lcc}
\hline Nodes & $\begin{array}{c}\text { Joint stiffness } \\
(\mathrm{rad})\end{array}$ & $\begin{array}{c}\text { Relative } \\
\text { difference }(\%)\end{array}$ \\
\hline 5,610 & $1.4589 \times 10^{-8}$ & \\
8,615 & $1.7103 \times 10^{-8}$ & 14.7 \\
11,027 & $1.9872 \times 10^{-8}$ & 13.94 \\
24,652 & $2.3715 \times 10^{-8}$ & 16.21 \\
28,668 & $2.3495 \times 10^{-8}$ & -0.94 \\
31,589 & $2.3877 \times 10^{-8}$ & 1.6 \\
38,629 & $2.4344 \times 10^{-8}$ & 1.92 \\
\hline
\end{tabular}




\begin{tabular}{ccc} 
TABLE 2: & Convergence of shell model results. \\
\hline Nodes & $\begin{array}{c}\text { Joint stiffness } \\
(\mathrm{rad})\end{array}$ & $\begin{array}{c}\text { Relative } \\
\text { difference }(\%)\end{array}$ \\
\hline 5,468 & $3.0349 \times 10^{-8}$ & \\
6,147 & $3.0387 \times 10^{-8}$ & 0.123 \\
19,125 & $3.0412 \times 10^{-8}$ & 0.0847 \\
72,110 & $3.0418 \times 10^{-8}$ & 0.0196 \\
\hline
\end{tabular}

This model produced a fairly good convergence, but the model was considered to be an unreasonable simplification of the real T-joint. In the real model there would be no gap, and it was unclear whether or not the welds were being represented accurately. Typically, spot welds perform two functions: they provide a physical fusion between the connected parts; and also increase the contact pressure. Having the welds represented in this way performed satisfactorily for the first function but not the second.

The second model, called the shell model, was used to replace the single solid model with a pair of equivalent surfaces representing each plate. This model was meshed using an 8 node (4 corner and 4 mid-nodes) shell element which had 6 degrees of freedom at each node. Figure 2 shows this mesh had excellent convergence when the mesh density was refined. Four simulations were run for shell model as shown in Table 2. By increasing the number of nodes by the factor of 15 resulted in variations in joint stiffness of $0.02 \%$. Thus this model with 5468 nodes is quite accurate.

In the third and final model, called the FE model (Finite Element model), there was no gap between the upper and lower plates, and the weld nuggets were represented as separate solids piercing the plates, following Kuo et al. [5].

This FE model allowed for a realistic representation of each spot weld as a nugget of fused metal which constrains the two parts to be in contact with each other with stress flowing only through the weld itself. The results are given in Tables 3 and 4. 
TABLE 3: Mesh refinement results

\begin{tabular}{lccc}
\hline Nodes & $\begin{array}{c}\text { Joint stiffness } \\
(\mathrm{rad})\end{array}$ & $\begin{array}{c}\text { Relative } \\
\text { error (\%) }\end{array}$ & $\begin{array}{c}\text { Significant } \\
\text { figures }\end{array}$ \\
\hline 6782 & $1.4516 \times 10^{-4}$ & 12.507 & 1 \\
10,337 & $1.4948 \times 10^{-4}$ & 9.9075 & 1 \\
12,524 & $1.5469 \times 10^{-4}$ & 6.7677 & 1 \\
32,466 & $1.6429 \times 10^{-4}$ & 0.9832 & 2 \\
40,064 & $1.6577 \times 10^{-4}$ & 0.0884 & 3 \\
44,001 & $1.6592 \times 10^{-4}$ & - & at least 3 \\
\hline
\end{tabular}

\section{Validation}

The main techniques used to validate our finding are accuracy and resolution checks. Rigorous validation is essential to ensure that experimental results are not misleading. When carrying out physical experimentation all results must be qualified with estimation of errors and we should practice similarly appropriate caution when carrying out computer simulations.

Accuracy: the degree to which the model represents reality.

Resolution: the degree to which the model distinguishes between design alternatives.

The FE model with 32,466 nodes was deemed to be sufficiently accurate for the analysis carried out in this work. At this mesh density, Table 3 shows the relative error falls below $1 \%$. All subsequent simulations were carried out using this mesh density and have an approximate error of $1 \%$. The resolution was calculated out by decreasing $V_{*}$ in four steps $(0.95,0.9,0.8$ and 0.5$)$ and the joint stiffness was calculated. Even a 0.05 change in $V_{*}$ affected the joint stiffness - see 'Relative difference (\%)' in Table 4. 


\begin{tabular}{ccc} 
TABLE 4: & Resolution test results for FE mo \\
\hline$V_{*}$ & $\begin{array}{c}\text { Joint stiffness } \\
(\mathrm{rad})\end{array}$ & $\begin{array}{c}\text { Relative } \\
\text { difference }(\%)\end{array}$ \\
\hline 1 & $1.6429 \times 10^{-4}$ & - \\
0.5 & $1.6398 \times 10^{-4}$ & 0.33 \\
0.8 & $1.6405 \times 10^{-4}$ & 0.14 \\
0.9 & $1.6402 \times 10^{-4}$ & 0.16 \\
0.95 & $1.6398 \times 10^{-4}$ & 0.19 \\
\hline
\end{tabular}

\subsection{Variation in joint stiffness}

Using stress concentration factor analysis, the stress concentration was found to be the highest for the welds nearest to T-joint intersection. The model described contains 13 spot welds, however it was decided on the basis of the highest stress concentration to analyse only welds $2,3,4,7,8,11 \& 12$ as shown in Figure 3, labelled clockwise from the top left of T-joint. In order to determine the variation in joint stiffness we first determine the input variable by running a Monte-Carlo simulation to generate a input data set and then running standard statistical analysis to determine the moments. The first six moments for the input variables of the simulation are listed in Table 5. The values contained in Table 6 represent the variation in joint stiffness using Six Sigma approach. See that the mean value of $1.6429 \times 10^{-4}$ is exactly the same as the base line value obtained using FE model as shown in Table 4.

\section{Conclusions}

We have used Six sigma approach to determine the stress value $\sigma$ of the input weld volume data $V_{*}$, Monte-Carlo simulations to determine the Moment of weld volume $V_{*}$ based on the stress value $\sigma$ gained from the use of Six Sigma approach and the Method of Moments and Finite Element to obtain joint 


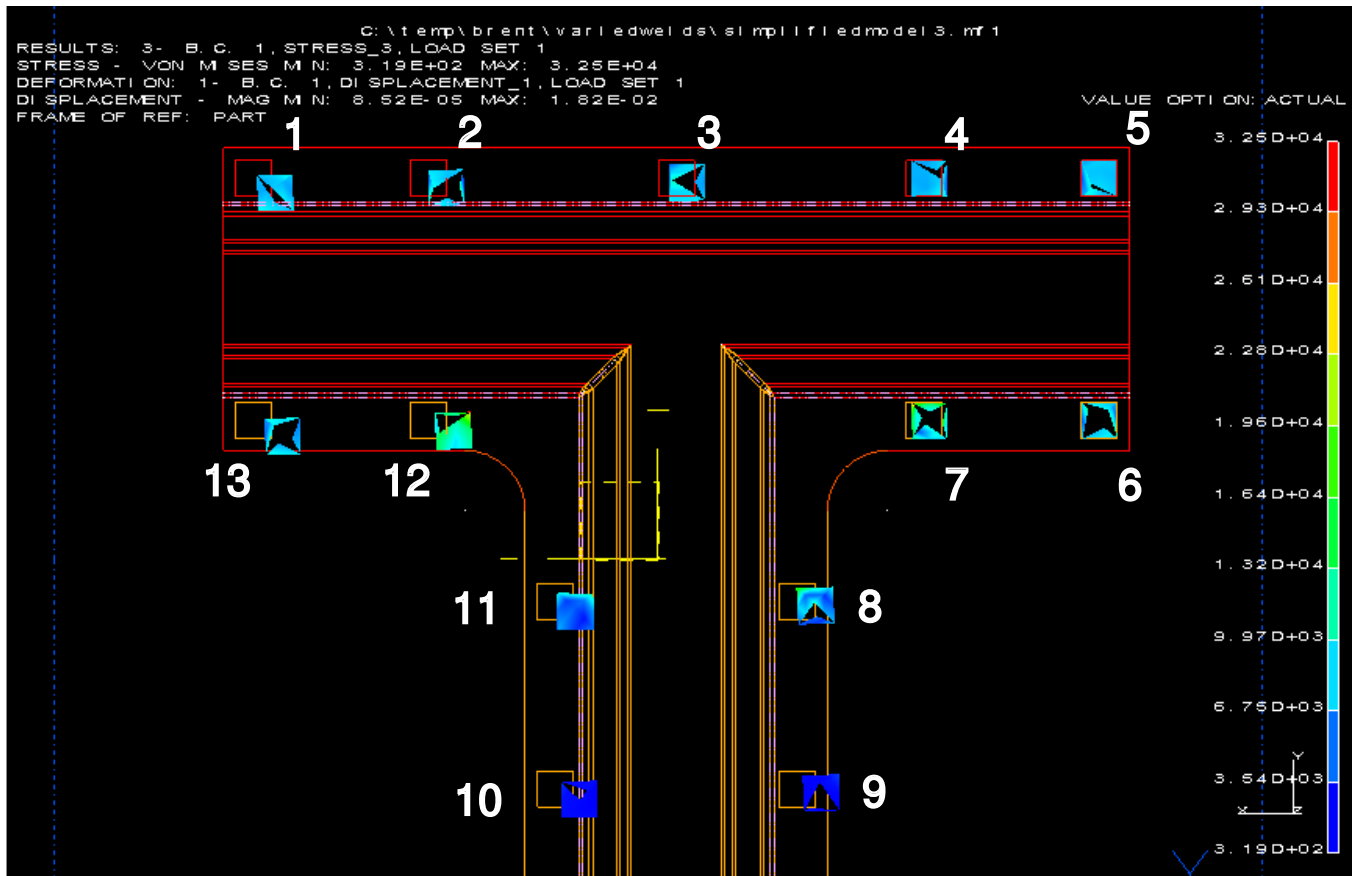

Figure 3: Weld locations, numbered clockwise from top left

TABle 5: Weld PDF moments (Six Sigma)

\begin{tabular}{lc}
\hline Moment & $V_{*}$ \\
\hline Mean & 1 \\
Variance & $1.1108 \times 10^{-3}$ \\
Skew & $-4.7511 \times 10^{-5}$ \\
Kurtosis & 2.9914 \\
$\mu_{5}$ & $-1.457 \times 10^{-3}$ \\
$\mu_{6}$ & 14.743 \\
\hline
\end{tabular}


TABLE 6: Joint stiffness variation (Six Sigma)

\begin{tabular}{lc}
\hline & Value $(\mathrm{rad})$ \\
\hline Approximation of mean & \\
First order & $1.6429 \times 10^{-4}$ \\
Second order & $1.6424 \times 10^{-4}$ \\
Approximation of variance & \\
First order & $3.7312 \times 10^{-14}$ \\
Second order & $3.8594 \times 10^{-5}$ \\
Third order & $3.8609 \times 10^{-5}$ \\
\hline
\end{tabular}

stiffness employing the Monte-Carlo data.

The Method of Moments is usually carried out on very simple applications, and our success in this limited study indicates that the method could be used on even more complicated applications.

\section{References}

[1] Bajaria H. J., Six sigma quality: points and counterpoints, Quality Australia, 15, pp.15-17, 2000. C572

[2] Beer F. P., Johnston E. R., \& Dewolf J. T., Mechanics of materials, McGraw-Hill, NY, 3rd edition, 2002. C573

[3] Burden R. L. \& Faires J. D., Numerical Analysis, PWS publishing company, Boston, 4th edition, 1988. C574

[4] Fenton J., Vehicle layout and analysis, Mechanical Engineering Publications, London, 1980. C575, C577 
[5] Kuo E. Y., Jayasuriya A. M. M. \& Wung P., The effect of high mileage spot weld degradation on vehicle body joint stiffness, SAE paper 2001-01-0426, 2001. C573, C579

[6] Okabe A. and Tomioka N., Estimating each element of joint stiffness matrix using neural network-estimation from the rotation vector at end of jointed part, SAE paper 2001-01-3219, 2001. C574

[7] Smith P., Into Statistics, Springer-Verlag, Singapore, 1997.

[8] Song Y., Flanigan D., Rivard C., Shahidi B. and Nguyen C., A new FEA method for the evaluation of a body joint, SAE paper 2001-01-0758, 2001. C575

[9] Steele C., Probabilistic design section, Swinburne University of Technology, Faculty of Engineering and Industrial Sciences, Lecture notes, 2003. C573, C574 